\title{
A Comparison Study on Selective Traffic Models with Handoff Management Scheme for Wireless Mobile Network Infrastructure
}

\author{
Bis wajit Bhowmik \\ Member of IEEE, IACSIT, IAENG, PASS, IAOE, IAS, and UACEE \\ Department of Computer Science \& Engineering, Indian Institute of Technology Guwahati, India \\ Email: b.bhowmik@iitg.ernet.in; biswajitbhowmik@gmail.com
}

\begin{abstract}
Wireless and Mobile Network Infrastructure services generally examine how different infrastructure providers are positioning themselves to compete in wireless services. These services analyze the opportunities and challenges facing by the infrastructure providers selling to wireless and mobile network operators worldwide. The opportunities and challenges are the review of strategies, market positioning, and future direction in the market for wireless and mobile network infrastructure. The service covers all generations of wireless and mobile network infrastructure: $2 \mathrm{G}, 2.5 \mathrm{G}, 3 \mathrm{G}$, and $4 \mathrm{G}$ so on global in scope. Majority of the users are mobile while availing these services. Mobility is the most important feature of any wireless and mobile network. Usually, continuous service is achieved by supporting handoff from one cell to another. Poorly designed handoff schemes tend to generate very heavy signaling traffic and, thereby, lead to a dramatic decrease in quality of service. In this paper we make a comparison study on some selective traffic models with priority handoff scheme. The study shows how a handoff procedure has a significant impact on wireless mobile network performance on the basis of two primary criteria - Blocking probability of originating calls and the forced termination probability of ongoing calls. In addition to this the study shows arrival rate of handoff calls, and approximate nu mber of mobile users that get serviced by underlying base station.
\end{abstract}

Index Terms - Mobile Station, Base Station, Traffic Model, Arrival Rate, Blocking Probability, Call Blocking Rate

\section{Introduction}

The rapid growth in the demand for wireless services has led to an intense research effort to achieve an efficient use of the resources of wireless and mobile network infrastructure (WMNI). The channel (frequency, time slot, spreading code, or combination of them) is a limited resource for any wireless communications. The traffics (request and demand for services) are increasing day by day for any kind of wireless services. Different WMNI providers are positioning themselves to compete these services in different ways with the opportunities and challenges. These include a review of strategies, market positioning, and future direction in the market for the WMNI. The services cover all generations of the WMNI- 2G, 2.5G, $3 \mathrm{G}$, and $4 \mathrm{G}$ so on global in scope. A major challenge for them (s mooth services) is proper allocation of channels to these ever increasing traffics. The channel associated with a current connection served by a base station (BS) is changed while a call is in progress because majority of the users are mobile while availing these services. Therefore, mobility is then become most important feature of any wireless and mobile network (WMN). It is natural from a user's perspective that once a service provider starts providing services to users; their services must be uninterrupted anyhow. A mobile station (MS) moves through the coverage area (service area) of the underlying BS. Services however may be interrupted while a MS crosses the cell boundary (coverage area) of its current BS. But continuous service is achieved by supporting handoff (handover) from one cell to the next adjacent cell as MS moves through the coverage area of the underlying BS. The handoff algorithms as proposed by different researchers are able to determine the dynamics of these MSs which move through the WMN [1-15].

Several competent factors influence to occurring a handoff. The most significant factors are MS's mobility irrespective of the direction of its movements, and relative signal strength (RSS) of the BSs. They affect as follows. First, when a MS moves across a cell boundary from the serving $B S_{1}$ to another $B S_{2}$. Second is deterioration in quality of the received signal in the current channel. Movements of MSs in cells are shown in Fig. 1 [2][4-5][10][15-17]. 


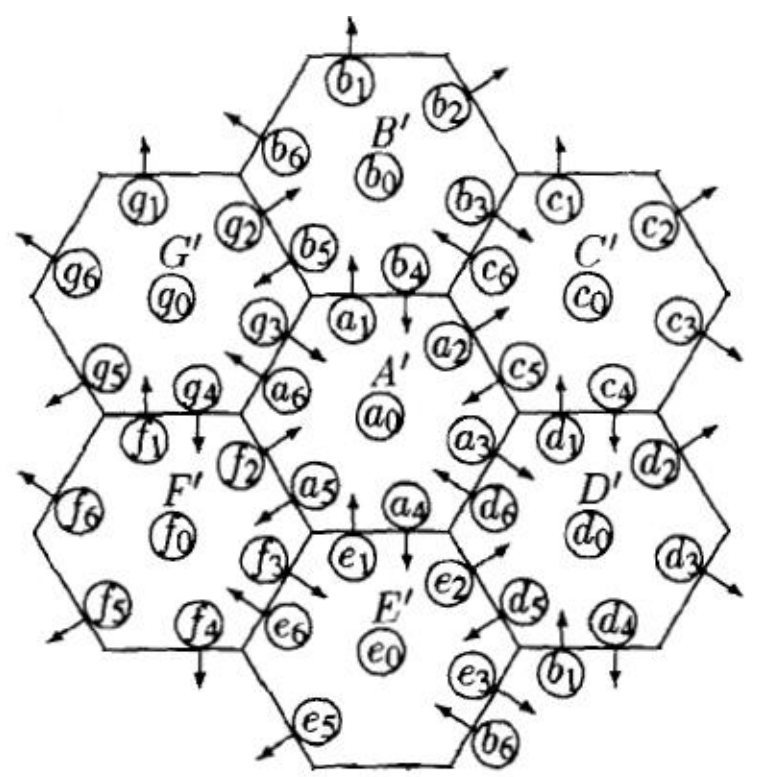

Fig. 1: Movements of the Mobile Stations

The handoff phenomena in a mobile network environment has become progressively more important issue as cell sizes shrink to accommodate an increasingly large MSs in terms of demand for services [4]. Although a handoff is divided into two broad categories - hard and soft handoffs, the term (handoff) we will use throughout this paper refers to former type of handoff (i.e. hard handoff). A schematic diagram of typical hard handoff is shown in Fig. 2.

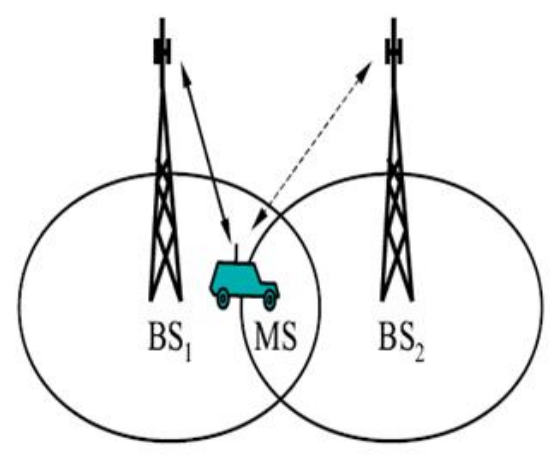

a. Before handoff

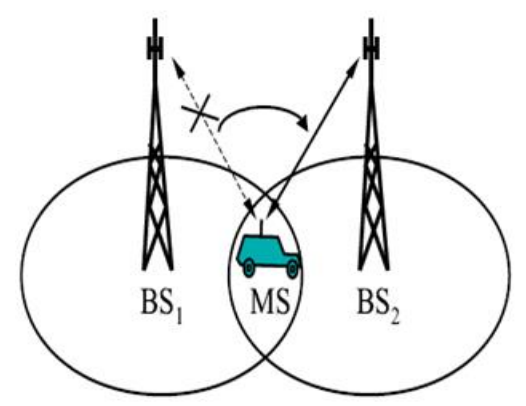

\section{b. After handoff}

Fig. 2: Hard handoff between the MS and BSs
In Fig. 2.a, we see that a MS is currently served by $B S_{1}$ and it is moving towards $B S_{2}$ with a sufficient RSS above its threshold [2] and without any handoff taken place. In the Fig. 2.b, we see that a MS has entered in a handoff region (a common coverage area of both $\mathrm{BS}_{1}$ and $\mathrm{BS}_{2}$ where the RSS from $B S_{1}$ is perhaps below its threshold and the RSS from $B S_{2}$ is perhaps above its threshold). At the moment services of the MS is just cut off from the $B S_{1}$ and is gained by the $B S_{2}$. Eventually, a handoff thus happened. In this case, the active set of MSs therefore consists of at most one BS at any given time. The cellular structure of the coverage area of a BS is shown in Fig. 3. The underlying BS is also known by a term mobile terminal (MT) [1$2][4][6][10][12]$ [18-19].

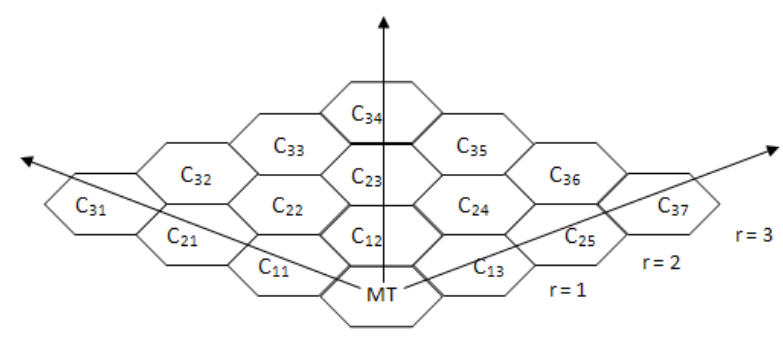

Fig. 3: Cellular structure of serving area of a BS

When a MS moves irrespective of a direction in a cell of the above cellular structure two cases and their corresponding sub cases may arise: (1) Call Acceptance - (a) A successful completion to the target cell, (b) New call arrival in the target cell, and (c) Active MT increases its resource demands. (2) Call Rejection - (a) A successful completion from the target cell. (b) Call termination in the target cell and (c) Active MT decreases its resource demands [1-2][17][19]. A poorly designed handoff scheme tends to a dramatic decrease in quality of services (QoS). The reason is that very heavy signaling traffic in particular is generated and a situation of more call rejection may be raised. Thus a handoff procedure has a significant impact on the performance of the system undersigned. A handoff scheme is generally designed based on traffic models with single or multiple traffics in a cell and with or without assigning any priority to a service request. In this paper we consider a priority based traffic models in presence of multiple traffics in a cell. For simplicity, we have selected two traffic models - El-Dolil et al's, and Xie and Kuek's models. The first model is single dimensional where as the second model is applicable for both single- and two-dimensional. This paper is organized as follows. First, we propose a suitable approach in section II. The approach is common for both the selected traffic models from the simulation point of view. The performance metrics of the selected traffic models are given in section III. The simulated results have been shown in section IV. At last, the paper is fin ished drawing remarkable conclusions on the performances measured for the traffic models selected [1-3][14]. 


\section{Proposed Approach}

Mobility is the most important feature of a wireless cellu lar mobile network. The MSs move randomly in the coverage area. Consider the Fig. 1 in this regard. But a handoff is only possible when a MS either crosses a cell boundary merely or its RSS is lower than threshold value. Crossing a cell boundary may be possible three ways. First, when MSs move from a cell in radial distance $r$ to a cell at radial distance $(r+1)$ and so on. Second, this case is exactly opposite to the previous one that means MSs move from a cell in radial distance $r$ to a cell at radial distance and so on. Third, $M S S$ in this case move from a cell $(r-1)$ to another along the same radial distance $r$. However, third case is very less responsible for a handoff to be happened since RSS of a MS remains same. Consider the Fig. 3 for the purpose. Second case is however comparatively less responsible for a handoff to be happened since RSS of a MS become strengthen as they move towards their servicing MT. First two cases have been shown in Fig. 4(a) and Fig. 4(b) respectively that happen in a cell of Figure 3. The cells A, X, Y....represent any cell $C_{r j}$ of the cellular structure shown in Fig. 3 [1-3].

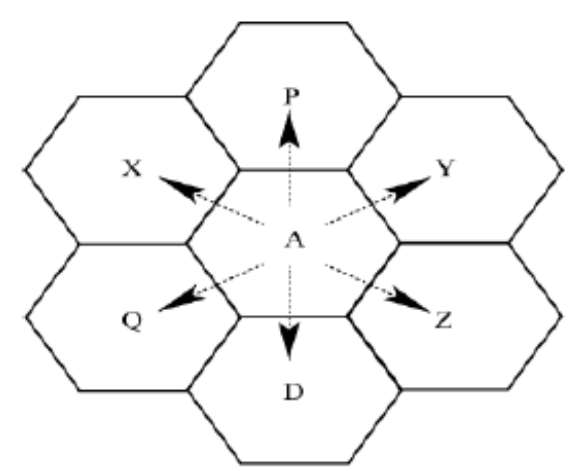

(a)

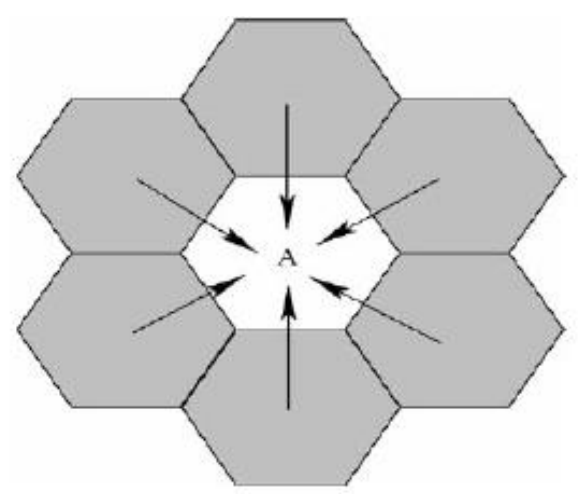

(b)

Fig. 4: Mobility of Mobile Stations

Now suppose that MSs move away from their current MT. Some of them may be come back to the previous redial level. Effective number of MSs those are actually would be handed-off will be less. Assume that this number is two-third of the MSs initiated requests. We exploit this feature to compute all the effective parameters of the selected traffic models and the associates that have been considered in this paper and in [1-2]. Therefore, handoff problem arises in cellular mobile and wireless networks when a communicating platform moves from a spatial (source) region served by one wireless gateway to a (target) region served by another [1-3][28]. This approach thus provides high precise location and tracking of MSs by exploiting these advanced traffic models.

\section{Proposed Approach}

It is important to ensure the MSs that today's WMNs even in next-generation would guarantee on the services of their requests. Therefore a handoff procedure would have a significant impact on WMNs performance. A proper traffic model is then required to effectively manage ever increasing traffics (MSs) serving by their respective MTs. The performance metrics (simulation parameters) responsible for providing better services by WMNs are organized as follows. (1) Two selected traffic models are presented to determine arrival rate $\left(\lambda_{H}\right)$ of handoff calls. (2) We see how blocking probability $\left(B_{O}\right)$ of originating calls and blocking probability $\left(B_{H}\right)$ of handoff calls are determined. (3) Next we see how call blocking rate $(\mathrm{CBR})$ is determined [1-2][9-10].

\subsection{Selection of Traffic Models}

For the WMNs, it is important to establish a traffic model before analyzing the performance of the system. Several traffic models have been established based on different assumptions such as user's mobility and the types of traffics. The traffics in telecommunication networks (TNs) and WMNs are traditionally classified into voice and data but the present rapid advancements of technology growth promise audio and video services the channels too. Here we consider two traffic models. First model is one-dimensional where as second model is both one- and second-dimensional. The dimension stands for either text or voice requests [3][29].

1) El-Dolil et al.'s Traffic Model: The highway is segmented into cellular structures (microcells) with small BSs. Assume that along the highway radio signals from mobile stations are cigar-shaped. With these assumptions, the $\lambda_{H}$ is determined as in [2-3][20] and is shown in (1) below.

$$
\lambda_{H}=\left(R_{c j}+R_{s h}\right) P_{h i}+R_{s h} P_{h h}
$$

Where,

$$
R_{c j}=\text { average rate of total calls carried in cell } j \text {. }
$$


$R_{s h}=$ the rate of success ful handoffs.

$P_{h i}=$ the probability that a $M S$ needs an handoff in cell $i$.

$P_{h h}=$ probability that a call that has already been handed off successfully would require another handoff .

We will exploit the estimate of general mathematical expressions for the parameters of (1) as estimated in [[1-3].

2) Xie and Kuek's Ttraffic Model: This model assumes a uniform density of mobile users (MSs) throughout an area of coverage of MTS and that a user is equally likely to move in any direction with respect to the cell boundary. The Figures 1, 4, and 5 illustrate distributions and mobility of MSs and their supporting MTs [21].

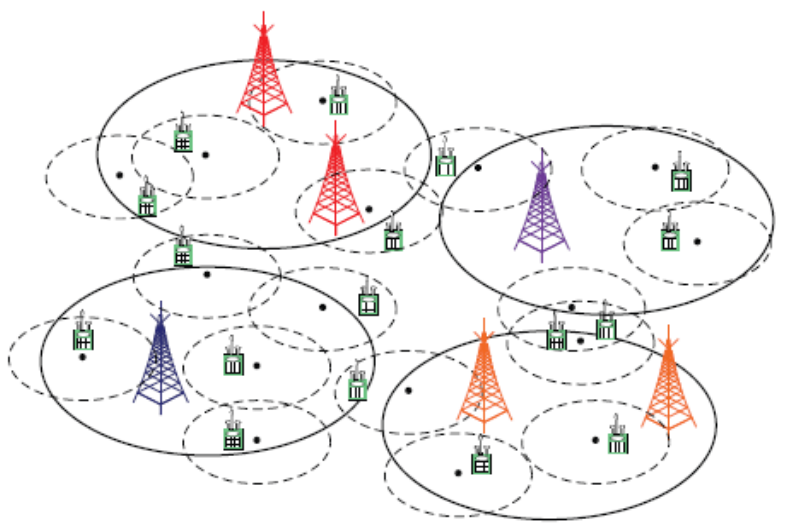

Fig. 5: Distribution and mobility of MSs

The $\lambda_{H}$ of this traffic model is determined with the above mentioned assumptions and is shown in (2) similar to [1][3][22].

$$
\lambda_{H}=\prod(E[c], \mu c-d w e l l)
$$

Where,

$E[c]=$ the average number of calls in a cell.

$\mu_{c-d w e l l}=$ the outgoing rate of MSs.

The mathematical formulations of all the simulation parameters $R_{c j}, R_{s h}, P_{h i}, P_{h h}, E[c]$, and $\mu_{c-d w e l l}$ to determine $\lambda_{H}$ of the model for (1) and (2) have been computed in [1-2]. Here we have simply reused them and their values for simulations.

\subsection{Determination of Blocking Probabilities}

A handoff request is generated in a cell when a MS approaches a cell from a neighboring cell with significant received signal strength. A priority is set to handoff requests by assigning $S_{R}$ channels exclusively for handoff calls out of $S$ channels assigned for a cell. The BSs communicate with MSs allocating a channel out of the channel band. Both originating and handoff calls share the remaining $S_{c}=S-S_{R}$ channels in the band. The channels sharing are shown in Fig. 6 [1-4]. The $\mu$ represents departure rate (number of MSs get serviced in unit time).

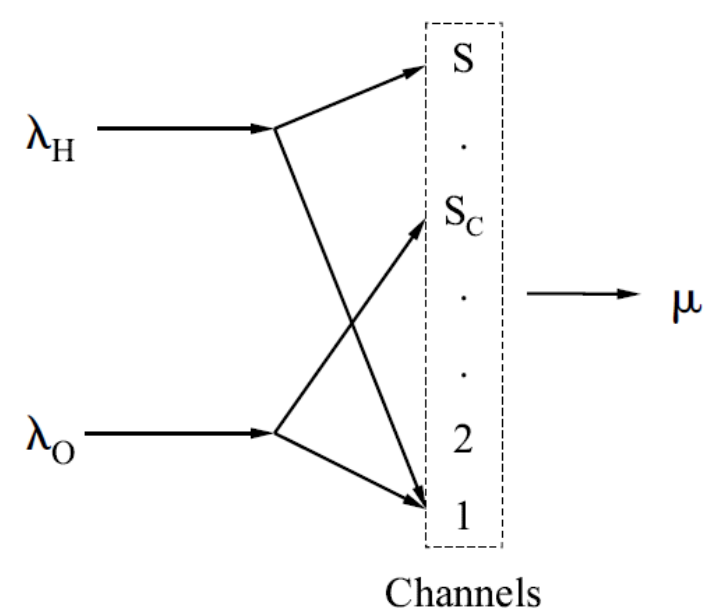

Fig. 6: System model with priority for handoff call

Two blocking probabilities $-B_{O}$ for orig inating calls and $B_{H}$ for handoff requests could be determined by (3) and (4) respectively similar to[3].

$$
B_{O}=\sum_{i=S_{C}}^{S} P(i)
$$

And,

$$
B_{H}=\frac{\left(\lambda_{O}+\lambda_{H}\right)^{S_{C}} \lambda_{H}^{S-S_{C}}}{S ! \mu^{S}} P(0)
$$

Here $P(0)$ and $P(i)$ are the steady-state probabilities at the states " 0 " and " $i$ " respectively [16][18][23].

\subsection{Determination of Call Blocking Rate}

A MS when initiates a call, it generally expects to be get serviced immed iately. Before that, a request must be enqueed in a priority queue. We take advantages of Splay operations performed on a splay tree once a call is scheduled. The priority queue generated in [5] is implemented to a splay tree. A call which is currently the root node of this splay tree gets served by its current $\operatorname{MT}\left(s a y, B S_{1}\right)$. A call is also scheduled from the tree using SIRO queuing principle. At a particular radial level $r$ from $M T$ as shown in Fig. 3 and at a specific time instant number of calls blocked (enqueed) for 
availing services i.e. call blocking rate $\left(C B R_{r}\right)$ [23] could be decided by (5) and (6) respectively.

$$
C B R_{r}=\frac{1}{3} \prod\left(R_{c_{r}}, B_{O},(2 r+1)\right), \mathrm{r}=1,2 \ldots . . \mathrm{R}_{\max }
$$

And,

$$
C B R_{r}=\frac{1}{3} \prod\left(R_{C_{j r}}, \quad B_{O},(2 \times r+1)\right)
$$

A well established WMN would generally provide $C B R_{r}$ that is expected to be $90-100 \%$ of the requests made by the MSs [1-2][5][19][23-25].

\section{Simulation}

The parameters of all the equations in this paper are used for simulation to analyze performances of any WMN for MSs having handoff behavior. Simu lations of the selected traffic models have been carried out in MATLAB Version 7.6.0.324 (R2008A). Numerical values of the fundamental parameters for handoff initiations, arrival rate $\lambda_{o}$ of originating calls, and service rate $\mu$ of the mobile users are based on COAI Report [26-27] developed for our beloved Megacity Kolkata. These parameters are set as $\lambda_{o}=1991$, and $\mu=2212$ [5]. We make use of these $\lambda_{o}, \mu$ and the numerical values of all the parameters (as computed in [1-2]) of the (1) through (6). We assume the shadow fading effect $\zeta(\mathrm{r})$ is $\log (\mathrm{r})$ in all the cases. We also suppose that a $M S$ makes at least 5 requests per day. The comparative performances of the selected traffic models in terms of the $B_{O}$, the $B_{H}$, and the $C B R_{r}$ are shown in Fig. 7, Fig. 8, Fig. 9, and Fig. 10 respectively [1-2][5][23-27].

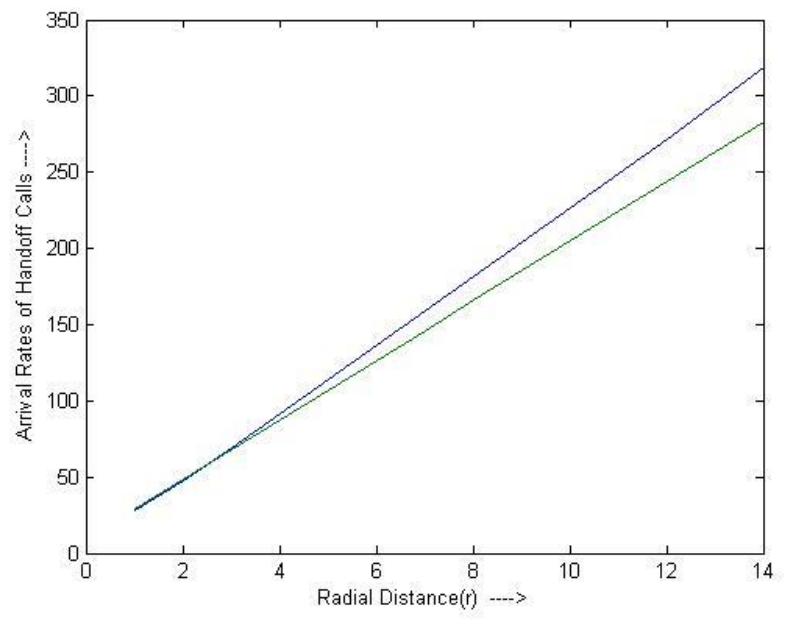

Fig. 7: Level-wise handoff requests of mobile users

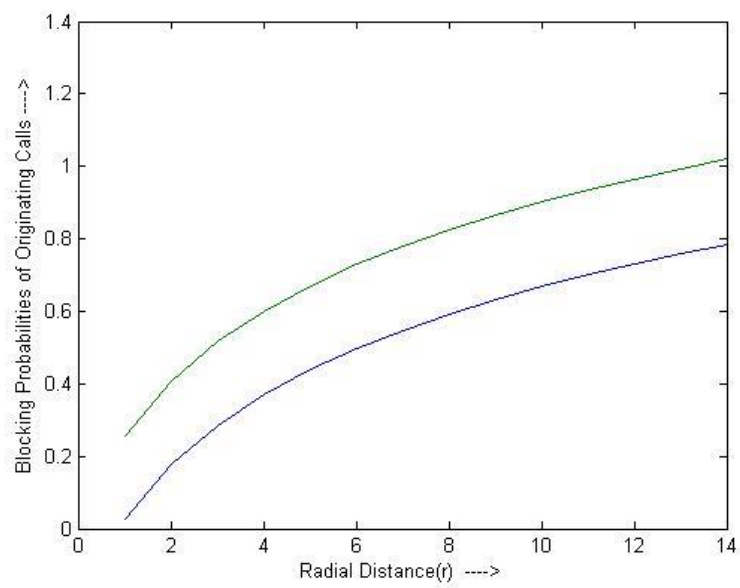

Fig. 8: Level-wise originated call requests of mobile users

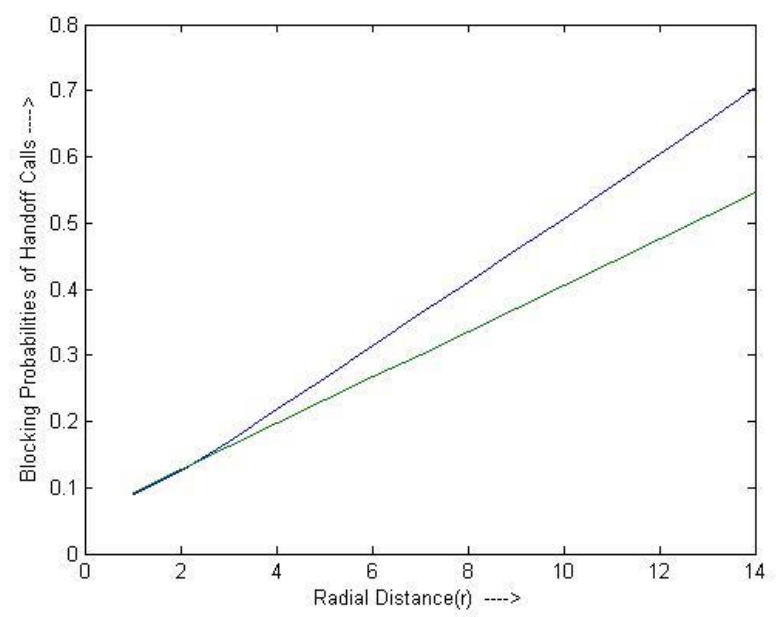

Fig. 9: Level-wise handed-off calls of mobile users

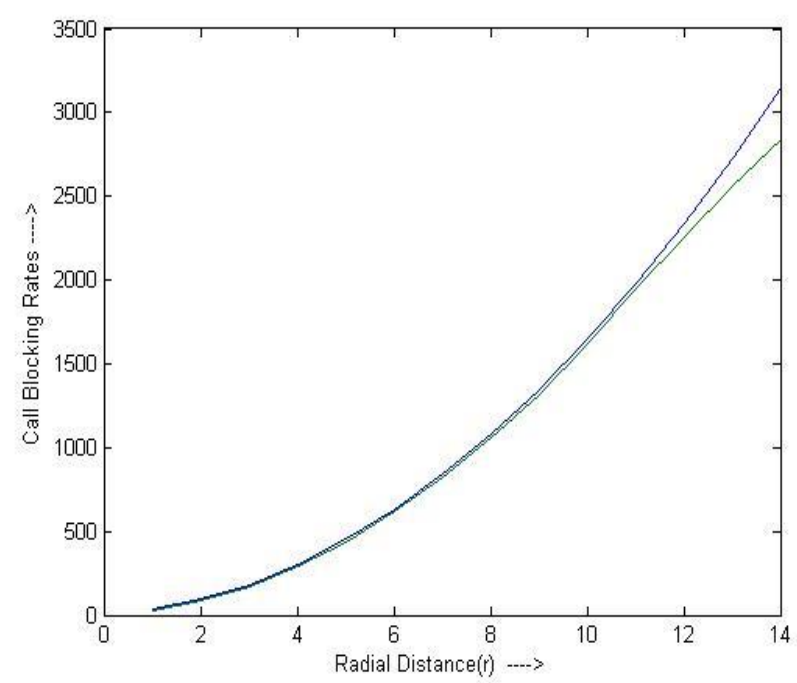

Fig. 10: CBR for selected traffic models

The blue and green color curves in the figures 7 through 10 are used to represent performance behaviors of the El-Dolil et al's, and Xie and Kuek's traffic models [1-3]. 


\section{Conclusion}

To the best of our knowledge, it is the first time that explicit mathematical expressions have been proposed to determine the arrival rate $\lambda_{H}$ of the handoff calls. We follow easy methods that are capable of implementing priority scheme analytically for the selected traffic models. Simu lation results show that our algorith $m$ performs better than some existing algorith ms. The proposed models have achieved expected number of handoffs on average. Compared with other handoff algorith ms, the only overhead of the proposed approach is proper allocation of channels. It is observed that increasing average number of calls of a mobile users in a day helps in improvement of the blocking probabilities $B_{O}$ and $B_{H}$. And these values are little deferred from their actual values. Another satisfactory achievement of the selected models is the working out of the rate of blocked calls. Finally, a crucial conclusion can be drawn that the graphical results ensure that the first traffic model works better than the second model except for the $B_{O}$. On the other hand the El-Dolil et al's model is limited in its applications as the model is one-dimensional. Whereas the Xie and Kuek's model is widely applicable for both one- and twodimensional data- text and/orvoice, audio and/orvideo.

\section{Acknowledgments}

The author acknowledges his heartily and warm gratitude to Pooja, Piyali Sarkar, and Nupur Thakur for their enthusiastic, continuous supports to complete this article in time and bring it to the level of publication.

\section{References}

[1] Biswajit Bhowmik, Pooja, Nupur Thakur, Piyali Sarkar, "Experimental Analys is of Xie and Kuek's Traffic Model with Handoff Scheme in Wireless Networks", IJIEEB, Vol. 4, No. 1, 2012. Pp: 34 43.

[2] Biswajit Bhowmik, Pooja, Piyali Sarkar, Nupur Thakur, "Modeling Prioritized Hard Handoff Management Scheme for Wireless Mobile Networks", IJCNIS, Vol. 4, No. 8, 2012 . Pp: 21 32.

[3] Ivan Stojmenovic, Qing-an Zeng and Dharma P. Agrawal, Handbook of Wireless Networks and Mobile Computing, John Wiley \& Sons, Second Edition, 2002.

[4] Biswajit Bhowmik, Smita Roy, Parag Kumar Guha Thakurta, Arnab Sarkar, "Priority Based Hard Handoff Management Scheme for Minimizing Congestion Control in Single Traffic Wireless
Mobile Networks", IJoAT, Vol. 2 No. 1, 2011. Pp: $90-99$.

[5] Biswajit Bhowmik, Pooja, Piyali Sarkar, Nupur Thakur, "Received Signal Strength Based Effective Call Scheduling in Wireless Mobile Network", IJoAT, Vo1. 2 No. 2, 2011. Pp: 292 305.

[6] Alexe E. Leu, Brian L. Mark, “A Discrete-Time Approach to Analyze Hard Handoff Performance in Cellular Networks", IEEE Transactions on Wireless Communications, Vol. 3, No. 5, 2004, Pp: $1721-1733$.

[7] S. A. Mawjoud, "Simulation of Handoff Techniques in Mobile Cellular Networks", A1Rafidain Engineering, Vol. 15 No.4, 2007, Pp: 31 -39 .

[8] N. Mohan, T. Ravichandran, "An Efficient Multiclass Call Admission Control and Adaptive Scheduling for WCDMA Wireless Network", European Journal of Scientific Research, Vol. 33, No. 4, 2009, Pp.718-727.

[9] Zhenqiang Ye et al, Predictive channel reservation for handoff prioritization in wireless cellular networks, Elsevier, Computer Networks, 51 (2007), Pp: $798-822$.

[10] Huamin Zhu and Kyung Sup Kwak, An Adaptive Hard Handoff Algorithm for Mobile Cellular Communication Systems, ETRI Journal, Vol. 28, No. 5, 2006, Pp: $676-679$.

[11] Biswajit Bhowmik, Pooja, Piyali Sarkar, Nupur Thakur, "Experimental Analysis of Single Traffic Priority Handoff Management Scheme and Signal Strength Based Effective Call Scheduling in Wireless Mobile Networks", UG Final Year Project Report, BCET, Durgapur, India, 2011. Available:www.bis wajitrbhow mik.co.nr/acad/proj.

[12] Zhenqiang Ye et al, Predictive channel reservation for handoff prioritization in wireless cellular networks, Elsevier, Computer Networks, Vol. 51, 2007. Pp: 798 - 822 .

[13] Kishor S. Trivedi, S. Dharmaraja 1, Xiaomin Ma, Analytic modeling of handoffs in wireless cellular networks, Elsevier, Information Sciences 148, 2002. Pp: $155-166$

[14] Wireless and Mobile Network InfrastructureAn Idc Continuous Intelligence Service. Available: http://www.idc.com

[15] Sanjay Dhar Roy, "A Timer based Handoff Algorithm for Multi-cellular Systems", ICETET 2008, IEEEXplore, Pp: 819 - 822.

[16] Hua Jiang and Stephen S. Rappaport, "Hand-Off Analysis for CBWL Schemes in Cellular Communications", CEAS Technical Report, No. 683, 1993. 
[17] Biswajit Bhowmik, Arnab Sarkar, Parag Kumar Guha Thakurta, "Simulation of Handoff Management Scheme for Improved Priority Based Call Scheduling with a Single Traffic System in Mobile Network", IJARCS, Vol. 1 No. 3, 2010. Pp: $354-358$.

[18] J K Sharma. "Operations Research - Theory and Application”, Macmillan Publishers, 3/e, 2006.

[19] S. A. El-Dolil, W. C. Wong, and R. Steele, Teletraffic performance of highway microcells with overlay macrocell, IEEE J. Select. Areas in Commun., Vol. 7, No. 1, 1989. Pp. 71-78.

[20] Wentao Huang ; Xinbing Wang ; Xiaojun Lin , Multicast capacity in mobile wireless ad hoc network with infrastructure support INFOCOM, 2012 Proceedings IEEEXplore, Pp:271 - 279.

[21] H. Xie and S. Kuek Priority handoff analysis, Proc. IEEE VTC '93, 1993. Pp. 855-858.

[22] P. K. Guha Thakurta, Souvik Sonar, Biswajit Bhowmik, Swapan Bhattacharya, Subhansu Bandyopadhyay, "A New Approach on Priority Queue based Scheduling with Handoff Management for Mobile Networks", 19th Int. Conf. on SEDE 2010, ISCA, California, USA. Pp: 69-74.

[23] Thomas H. Cormen, Charles E. Leiserson, Ronald L. Rivest, and Clifford Stein, Introduction to Algorithms, PHI, 2nd Edition, 2006.

[24] D. Samanta, Classic Data Structures, PHI, 2nd Edition, $18^{\text {th }}$ printing, 2010.

[25] Biswajit R Bhowmik, Design and Analysis of Algorithms, S.K. Kataria \& Sons, Second Edition, 2012.

[26] COAI Annual Report 2010-11. Available: http://www.coai.com/study_papers.php?val=2010.

[27] Urban structure of Kolkata. Available: http://en.wikipedia.org/wiki/Kolkata\#Geography.

[28] Jo“rg Diedericha, Martina Zitterbart, A simple and scalable handoff prioritization scheme, Elsevier, Computer Communications 28, 2005. Pp: 773-789.

[29] Fotini-Niovi Pavlidou, "Two-Dimensional Traffic Models for Cellular Mobile Systems", IEEE Transactions on Communications, Vol. 42, No. 2/3/4, 1994. Pp: 1505-1511.

Biswajit Bhowmik is currently doing $\mathrm{PhD}$ in the Department of Computer Science \& Engineering of Indian Institute of Technology Guwahati. He was a very renowned faculty member with Bengal College of Engineering and Technology, Durgapur, India as Assistant Professor in the Department of Computer Science \& Engineering for almost 7 years. He is a me mber of different professional bodies such as IEEE, IACSIT, IAENG, IAS, IAOE, ISOC, PASS, UACEE etc. He is also a member of some professional societies such as IEEE Computer Society, IEEE Communications Societies, IAENG Society of Computer Science, IAENG Society of W ireless Networks, IAENG Society of Software Engineering, and IAENG Society of Artificial Intelligence. $\mathrm{He}$ is reviewer of several international journals such as ETASR, IJCSIC, IJCSIS, JACSM, IJoAT, JWMC etc in the area of computer science, wireless networks, and mobile computing. He has authored a book titled Design and Analysis of Algorithms. He has many publications in international journals including international conference proceedings on the subjects ranging from Algorithms Analysis, Graph Theory, Wireless Networks, and Mobile Computing. In addition his area of interests includes Data Structures \& Algorithms, Computational Geometry, and Green Computing. He has guided several projects at under graduate level.

How to cite this paper: Biswajit Bhowmik,"A Comparison Study on Selective Traffic Models with Handoff Management Scheme for Wireless Mobile Network Infrastructure", International Journal of Information Technology and Computer Science(IJITCS), vol.5, no.2, pp.66-72, 2013.DOI: 10.5815/ijitcs.2013.02.07 